\title{
SOME EQUALITIES FOR CONTINUED FRACTIONS OF GENERALIZED ROGERS-RAMANUJAN TYPE
}

\author{
YongQun Li AND Xiantao WANG
}

\begin{abstract}
In this paper, we first discuss the convergence of the continued fractions of generalized Rogers-Ramanujan type in the modified sense. Then we prove several equalities concerning these continued fractions. The proofs of our main results are mainly based on the Bauer-Muir transformation.
\end{abstract}

\section{Preliminary material}

If the sequence $\left\{S_{n}(0)\right\}$ of the approximants of the continued fraction $b_{0}+$ $K\left(a_{n} / b_{n}\right)$ converges to a point $f$ in the extended complex plane $\overline{\mathbb{C}}=\mathbb{C} \cup\{\infty\}$, then we call that the continued fraction $b_{0}+K\left(a_{n} / b_{n}\right)$ converges to $f$ in the classical sense, and write

$$
b_{0}+K\left(a_{n} / b_{n}\right)=f,
$$

where

$$
S_{n}(0)=b_{0}+\frac{a_{1}}{b_{1}}+\frac{a_{2}}{b_{2}}+\frac{a_{3}}{b_{3}}+\cdots+\frac{a_{n}}{b_{n}} .
$$

Two continued fractions $b_{0}+K\left(a_{n} / b_{n}\right)$ and $d_{0}+K\left(c_{n} / d_{n}\right)$ are equivalent if they have the same sequence of classical approximants. The following result is from [6] or [8].

Proposition 1.1. Continued fractions $b_{0}+K\left(a_{n} / b_{n}\right)$ and $d_{0}+K\left(c_{n} / d_{n}\right)$ are equivalent if and only if there exists a sequence of non-zero constants $\left\{r_{n}\right\}$ with $r_{0}=1$ such that $c_{n}=r_{n} r_{n-1} a_{n}(n=1,2, \ldots)$ and $d_{n}=r_{n} b_{n}(n=0,1, \ldots)$.

As in [5] or [8], we introduce the following definition.

Received February 6, 2007; Revised October 26, 2009.

2010 Mathematics Subject Classification. Primary 40A15; Secondary 11J70.

Key words and phrases. Bauer-Muir transformation, convergence in the modified sense, convergence in the classical sense, equality, continued fraction of generalized RogersRamanujan type.

The research was partly supported by NSF of China (No. 11071063) and Academic project for youth from Chinese government. 
Definition 1.2. The Bauer-Muir transform of a continued fractions $b_{0}+$ $K\left(a_{n} / b_{n}\right)$ with respect to a sequence $\left\{\omega_{n}\right\}$ from $\mathbb{C}$ is the continued fraction $d_{0}+K\left(c_{n} / d_{n}\right)$ whose $n$th numerators $C_{n}$ and denominators $D_{n}$ are given by

$$
\begin{array}{ll}
C_{-1}=1, & D_{-1}=0, \\
C_{n}=A_{n}+A_{n-1} \omega_{n}, & D_{n}=B_{n}+B_{n-1} \omega_{n}
\end{array}
$$

for $n=0,1,2, \ldots$, where $\left\{A_{n}\right\}$ and $\left\{B_{n}\right\}$ are the $n$th numerators and denominators of $b_{0}+K\left(a_{n} / b_{n}\right)$.

Definition 1.3. If the Bauer-Muir transform $d_{0}+K\left(c_{n} / d_{n}\right)$ of a continued fraction $b_{0}+K\left(a_{n} / b_{n}\right)$ converges, then we say that the continued fractions $b_{0}+K\left(a_{n} / b_{n}\right)$ converges in the modified sense, and we write

$$
b_{0}+K\left(a_{n} / b_{n}\right)=d_{0}+K\left(c_{n} / d_{n}\right) \text { (m.c.). }
$$

In fact, what the Bauer-Muir transformation does is to give a continued fractions $d_{0}+K\left(c_{n} / d_{n}\right)$ whose classical approximants $T_{n}(0)$ are equal to the modified approximants $S_{n}\left(\omega_{n}\right)$ of $b_{0}+K\left(a_{n} / b_{n}\right)$. Therefore, if the BauerMuir transform $d_{0}+K\left(c_{n} / d_{n}\right)$ of a continued fraction $b_{0}+K\left(a_{n} / b_{n}\right)$ converges to $f^{*}=\lim _{n \rightarrow \infty} S_{n}\left(\omega_{n}\right)$, in the classical sense, then the continued fraction $b_{0}+K\left(a_{n} / b_{n}\right)$ converges to $f^{*}$ in the modified sense. Some caution is in order here, as noted by Thron and Waadeland in [10], if the modifying sequence $\left\{\omega_{n}\right\}$ can be arbitrary, almost anything can happen.

First, let's recall the following result from [8], which is crucial for the proofs of our main results.

Proposition 1.4. The Bauer-Muir transformation of a continued fraction $b_{0}+$ $K\left(a_{n} / b_{n}\right)$ exists with respect to the sequence of complex numbers $\left\{\omega_{n}\right\}(n=$ $0,1,2, \ldots)$ if and only if

$$
\lambda_{n}=a_{n}-\omega_{n-1}\left(b_{n}+\omega_{n}\right) \neq 0(n \geq 1) .
$$

If this Bauer-Muir transformation exists, then it is given by $d_{0}+K\left(c_{n} / d_{n}\right)$, where $d_{0}=b_{0}+\omega_{0}, d_{1}=b_{1}+\omega_{1}, d_{n}=b_{n}+\omega_{n}-\omega_{n-2} \lambda_{n} / \lambda_{n-1}$ for $n \geq 2$ and $c_{1}=\lambda_{1}, c_{n}=a_{n-1} \lambda_{n} / \lambda_{n-1}$ for $n \geq 2$.

A natural problem comes out: does a continued fraction converge in the modified sense although it is not convergent in the classic sense?

This problem has been considered by many authors. Among them, Alladi discussed this problem for continued fractions of modified Rogers-Ramanujan type in [1] and proved:

Theorem A. If $|q|<1$, then the continued fraction $q+K\left(1 / q^{2 n+1}\right)$, which is divergent in the classical sense, is convergent in the modified sense.

In [7], Lee and Sohn continued to study this problem and obtained:

Theorem B (Modified Rogers-Ramanujan continued fraction). If $|q|<1$, then the continued fraction $q+K\left(1 / q^{2 n+1}\right)$ converges in the modified sense, 
and

$$
q+\frac{1}{q^{3}}+\frac{1}{q^{5}}+\frac{1}{q^{7}}+\cdots=1+\frac{q}{1}+\frac{q^{2}}{1}+\frac{q^{3}}{1}+\frac{q^{4}}{1}+\ldots \text { (m.c.). }
$$

For a polynomial $p(x)$ in $\mathbb{C}[x]$, we introduce the following notation.

$$
E(p)=\{x \in \mathbb{C}: p(x)=0\} \cup\{0\} .
$$

The first aim of this paper is to discuss Theorem B further and get the following generalization.

Theorem 1.5. For any non-zero polynomial $a_{0}(x)$ in $\mathbb{C}[x]$, and for any fixed $x \in \mathbb{C}-E\left(a_{0}\right)$, if $|x|<1$, then the continued fraction

$$
k_{1} a_{0}+\frac{k_{2}^{2}}{k_{1} a_{1}}+\frac{k_{2}^{2}}{k_{1} a_{2}}+\frac{k_{2}^{2}}{k_{1} a_{3}}+\cdots
$$

which is divergent in the classical sense, converges in the modified sense, and

$$
\begin{aligned}
& k_{1} a_{0}+\frac{k_{2}^{2}}{k_{1} a_{1}}+\frac{k_{2}^{2}}{k_{1} a_{2}}+\frac{k_{2}^{2}}{k_{1} a_{3}}+\cdots \\
= & k_{2}+\frac{k_{1} k_{2} a_{0}}{k_{2}}+\frac{k_{2}^{2} x^{d}}{k_{2}}+\frac{k_{1} k_{2} a_{0} x^{d}}{k_{2}}+\frac{k_{2}^{2} x^{2 d}}{k_{2}}+ \\
& \frac{k_{1} k_{2} a_{0} x^{2 d}}{k_{2}}+\frac{k_{2}^{2} x^{3 d}}{k_{2}}+\frac{k_{1} k_{2} a_{0} x^{3 d}}{k_{2}}+\ldots
\end{aligned}
$$

where $k_{1}$ and $k_{2}$ are two non-zero constants, $a_{n}=a_{0} x^{n d}$ for $n \geq 1$ and $d$ is a positive constant.

Remark 1.6. We can get (1.2) by putting $k_{1}=k_{2}=1, a_{0}=q$ and $d=2$ in (1.3).

In [4], Berndt and Yee studied the continued fractions of generalized RogersRamanujan type and got the following equality.

Theorem C. For $|q|<1$,

$$
1-\frac{q}{1}+\frac{q}{1}-\frac{q}{1}+\frac{q}{1}-\frac{q^{2}}{1}+\frac{q^{2}}{1}-\frac{q^{2}}{1}+\frac{q^{2}}{1}-\ldots=\frac{1}{1}+\frac{q}{1}+\frac{q^{2}}{1}+\frac{q^{3}}{1}+\frac{q^{4}}{1}+\ldots
$$

As the second aim of this paper, we prove the following two theorems. The methods used in the proofs of these two theorems follow from [4].

Theorem 1.7. For $|q|<1$,

$$
1-\frac{q}{1}+\frac{q}{1}-\frac{q^{2}}{1}+\frac{q^{2}}{1}-\frac{q^{3}}{1}+\frac{q^{3}}{1}-\ldots=\frac{1}{1}+\frac{q}{1}+\frac{q^{3}}{1}+\frac{q^{5}}{1}+\frac{q^{7}}{1}+\ldots
$$


Theorem 1.8. For $|q|<1$,

$$
1+\frac{q}{1}-\frac{q}{1}+\frac{q^{2}}{1}-\frac{q^{2}}{1}+\frac{q^{3}}{1}-\frac{q^{3}}{1}+\ldots=\frac{1}{1}-\frac{q}{1}+\frac{q^{3}}{1}+\frac{q^{5}}{1}+\frac{q^{7}}{1}+\cdots
$$

As an application of Theorems 1.7 and 1.8 and Corollaries 3.1 and 3.4, we can easily get the following four equalities.

\section{Corollary 1.9 .}

(1.6)

$$
\begin{gathered}
\left(\frac{1}{1}-\frac{q}{1}+\frac{q}{1}-\frac{q^{2}}{1}+\frac{q^{2}}{1}-\frac{q^{3}}{1}+\frac{q^{3}}{1}-\ldots\right)+\left(\frac{1}{1}+\frac{q}{1}-\frac{q}{1}+\frac{q^{2}}{1}-\frac{q^{2}}{1}+\frac{q^{3}}{1}-\frac{q^{3}}{1}+\ldots\right)=2, \\
\left(\frac{1}{1}-\frac{a q}{1}+\frac{a q}{1}-\frac{a q^{k+1}}{1}+\frac{a q^{k+1}}{1}-\frac{a q^{2 k+1}}{1}+\frac{a q^{2 k+1}}{1}-\ldots\right) \\
(1.7)+\left(\frac{1}{1}+\frac{a q}{1}-\frac{a q}{1}+\frac{a q^{k+1}}{1}-\frac{a q^{k+1}}{1}+\frac{a q^{2 k+1}}{1}-\frac{a q^{2 k+1}}{1}+\ldots\right)=2,
\end{gathered}
$$

$$
\begin{gathered}
\left(\frac{1}{1}-\frac{q}{1}+\frac{q}{1}-\frac{q^{3}}{1}+\frac{q^{3}}{1}-\frac{q^{5}}{1}+\frac{q^{5}}{1}-\ldots\right)+\left(\frac{1}{1}+\frac{q}{1}-\frac{q}{1}+\frac{q^{3}}{1}-\frac{q^{3}}{1}+\frac{q^{5}}{1}-\frac{q^{5}}{1}+\ldots\right)=2, \\
\left(\frac{1}{1}-\frac{q}{1}+\frac{q}{1}-\frac{q^{k+1}}{1}+\frac{q^{k+1}}{1}-\frac{q^{2 k+1}}{1}+\frac{q^{2 k+1}}{1}-\ldots\right) \\
+\left(\frac{1}{1}+\frac{q}{1}-\frac{q}{1}+\frac{q^{k+1}}{1}-\frac{q^{k+1}}{1}+\frac{q^{2 k+1}}{1}-\frac{q^{2 k+1}}{1}+\ldots\right)=2 .
\end{gathered}
$$

On page 46 of Ramanujan's lost notebook [9], there is a theorem which is stated as follows (see also [2]) and was proved in [3].

Theorem D. Let $k \geq 0, \alpha=(1+\sqrt{1+4 k}) / 2$ and $\beta=(-1+\sqrt{1+4 k}) / 2$. Then, for $|q|<1$ and $\operatorname{Re}(q)>0$,

$$
1+\frac{k+q}{1}+\frac{k+q^{2}}{1}+\cdots=\alpha+\frac{q}{\alpha+\beta q}+\frac{q^{2}}{\alpha+\beta q^{2}}+\cdots
$$

In [7], Lee and Sohn obtained the following generalization of (1.10).

Theorem E. For a fixed natural number $r$, suppose that $d \geq 1$ and $m_{r} \geq$ $m_{r-1} \geq \cdots \geq m_{1} \geq 1$. Then we have

$$
k_{1}+\frac{k_{2}+a_{1}}{k_{1}}+\frac{k_{2}+a_{2}}{k_{1}}+\ldots=\alpha+\frac{a_{1}}{\alpha+\beta q^{d}}+\frac{a_{2}}{\alpha+\beta q^{2 d}}+\ldots,
$$

where

and

$$
a_{n}=q^{m_{1}+(n-1) d}+q^{m_{2}+(n-1) d}+\cdots+q^{m_{r}+(n-1) d}
$$

$$
\beta=\frac{-k_{1}+\sqrt{k_{1}^{2}+4 k_{2}}}{2}, \alpha=k_{1}+\beta .
$$


The last aim of this paper is to discuss Theorems D and E further. Our result is as follows.

Theorem 1.10. Let $a_{1}(x)$ be a non-zero polynomial in $\mathbb{C}[x]$. Then for any fixed $x$ in $\mathbb{C}-E\left(a_{1}\right)$, we have the following equality.

$$
k_{1}+\frac{k_{2}+a_{1}}{k_{1}}+\frac{k_{2}+a_{2}}{k_{1}}+\cdots=\alpha+\frac{a_{1}}{\alpha+\beta x^{d}}+\frac{a_{2}}{\alpha+\beta x^{2 d}}+\cdots,
$$

where $a_{n}=a_{1} x^{(n-1) d}$ for $n \geq 2, \beta=\left(-k_{1}+\sqrt{k_{1}^{2}+4 k_{2}}\right) / 2, \alpha=k_{1}+\beta$ and $d$ is a positive constant.

Remark 1.11. We can get (1.11) by putting $a_{1}(q)=q^{m_{1}}+q^{m_{2}}+\cdots+q^{m_{r}}$ in (1.12), and (1.10) by putting $k_{1}=1, k_{2}=k, a_{1}(q)=q$ and $d=1$.

\section{The proofs of Theorem 1.5 and its corollaries}

Proof of Theorem 1.5. We shall prove (1.3) from the left side to the right side by using the Bauer-Muir transformation. For convenience, we let

$$
R(x)=k_{1} a_{0}+\frac{k_{2}^{2}}{k_{1} a_{1}}+\frac{k_{2}^{2}}{k_{1} a_{2}}+\frac{k_{2}^{2}}{k_{1} a_{3}}+\cdots .
$$

We choose the modifying factors for $R(x)$ as follows:

$$
\omega_{i}^{(0)}=k_{2}-k_{1} a_{i}=k_{2}-k_{1} a_{0} x^{i d}, \quad i=0,1,2, \ldots
$$

Obviously, $\lambda_{i}^{(0)}=k_{1} k_{2} a_{0} x^{(i-1) d} \neq 0(i=1,2,3, \ldots)$. Then the Bauer-Muir transformation implies that

$$
\begin{aligned}
R(x) & =k_{2}+\frac{k_{1} k_{2} a_{0}}{k_{2}}+\frac{k_{2}^{2} x^{d}}{k_{2}-k_{2} x^{d}+k_{1} a_{0} x^{d}}+\frac{k_{2}^{2} x^{d}}{k_{2}-k_{2} x^{d}+k_{1} a_{0} x^{2 d}}+\cdots \\
& =k_{2}+\frac{k_{1} k_{2} a_{0}}{k_{2}}+\frac{k_{2}^{2} x^{d}}{R_{1}(x)}
\end{aligned}
$$

where

$$
R_{1}(x)=k_{2}-k_{2} x^{d}+k_{1} a_{0} x^{d}+\frac{k_{2}^{2} x^{d}}{k_{2}-k_{2} x^{d}+k_{1} a_{0} x^{2 d}}+\frac{k_{2}^{2} x^{d}}{k_{2}-k_{2} x^{d}+k_{1} a_{0} x^{3 d}}+\cdots .
$$

Now we choose the modifying factors for $R_{1}(x)$ as follows:

$$
\omega_{i}^{(1)}=k_{2} x^{d}-k_{1} a_{0} x^{(i+1) d}, \quad i=0,1,2, \ldots
$$

We easily know that $\lambda_{i}^{(1)}=k_{1} k_{2} a_{0} x^{i d} \neq 0(i=1,2,3, \ldots)$. By using the Bauer-Muir transformation once more, we have that

$$
\begin{aligned}
R_{1}(x) & =k_{2}+\frac{k_{1} k_{2} a_{0} x^{d}}{k_{2}}+\frac{k_{2}^{2} x^{2 d}}{k_{2}-k_{2} x^{2 d}+k_{1} a_{0} x^{2 d}}+\frac{k_{2}^{2} x^{2 d}}{k_{2}-k_{2} x^{2 d}+k_{1} a_{0} x^{3 d}}+\cdots \\
& =k_{2}+\frac{k_{1} k_{2} a_{0} x^{d}}{k_{2}}+\frac{k_{2}^{2} x^{2 d}}{R_{2}(x)}
\end{aligned}
$$


where

$$
\begin{aligned}
R_{2}(x)= & k_{2}-k_{2} x^{2 d}+k_{1} a_{0} x^{2 d} \\
& +\frac{k_{2}^{2} x^{2 d}}{k_{2}-k_{2} x^{2 d}+k_{1} a_{0} x^{3 d}}+\frac{k_{2}^{2} x^{2 d}}{k_{2}-k_{2} x^{2 d}+k_{1} a_{0} x^{4 d}}+\cdots
\end{aligned}
$$

For $j=2,3,4, \ldots$, we let

$$
\begin{aligned}
R_{j}(x)= & k_{2}-k_{2} x^{j d}+k_{1} a_{0} x^{j d}+\frac{k_{2}^{2} x^{j d}}{k_{2}-k_{2} x^{j d}+k_{1} a_{0} x^{(j+1) d}} \\
& +\frac{k_{2}^{2} x^{j d}}{k_{2}-k_{2} x^{j d}+k_{1} a_{0} x^{(j+2) d}}+\frac{k_{2}^{2} x^{j d}}{k_{2}-k_{2} x^{j d}+k_{1} a_{0} x^{(j+3) d}}+\cdots
\end{aligned}
$$

By applying the Bauer-Muir transformation to $R_{j}(x)(j=2,3,4, \ldots)$ and repeating the procedures as above, we find that, if we take $\omega_{i}^{(j)}=k_{2} x^{j d}-$ $k_{1} a_{0} x^{(j+i) d}(i=0,1,2, \ldots)$, then $\lambda_{i}^{(j)}=k_{1} k_{2} a_{0} x^{(i-1+j) d} \neq 0(i=1,2,3, \ldots)$. Similar discussions as above show that

$$
\begin{aligned}
R(x)= & k_{2}+\frac{k_{1} k_{2} a_{0}}{k_{2}}+\frac{k_{2}^{2} x^{d}}{k_{2}-k_{2} x^{d}+k_{1} a_{0} x^{d}}+\frac{k_{2}^{2} x^{d}}{k_{2}-k_{2} x^{d}+k_{1} a_{0} x^{2 d}}+\cdots \\
= & k_{2}+\frac{k_{1} k_{2} a_{0}}{k_{2}}+\frac{k_{2}^{2} x^{d}}{k_{2}}+\frac{k_{1} k_{2} a_{0} x^{d}}{k_{2}}+\frac{k_{2}^{2} x^{2 d}}{k_{2}-k_{2} x^{2 d}+k_{1} a_{0} x^{2 d}} \\
& +\frac{k_{2}^{2} x^{2 d}}{k_{2}-k_{2} x^{2 d}+k_{1} a_{0} x^{3 d}}+\cdots \\
= & \cdots \\
= & k_{2}+\frac{k_{1} k_{2} a_{0}}{k_{2}}+\frac{k_{2}^{2} x^{d}}{k_{2}}+\frac{k_{1} k_{2} a_{0} x^{d}}{k_{2}}+\frac{k_{2}^{2} x^{2 d}}{k_{2}}+\frac{k_{1} k_{2} a_{0} x^{2 d}}{k_{2}}+\cdots \\
& +\frac{k_{2}^{2} x^{(j+1) d}}{k_{2}-k_{2} x^{(j+1) d}+k_{1} a_{0} x^{(j+1) d}}+\frac{k_{2}^{2} x^{(j+1) d}}{k_{2}-k_{2} x^{(j+1) d}+k_{1} a_{0} x^{(j+2) d}}+\cdots
\end{aligned}
$$

By letting $j$ tend to $\infty$ in (2.1), we get (1.3).

The left side of (1.3) and the continued fraction $b_{0}^{*}+K\left(a_{n}^{*} / b_{n}^{*}\right)$ are equivalent, where $a_{n}^{*}=r_{n} r_{n-1} k_{2}^{2}$ for $n>0, b_{n}^{*}=r_{n} k_{1} a_{n}$ for $n \geq 0$, and $r_{0}=1, r_{n}=1 / k_{2}$ for $n \geq 1$. But the continued fraction $b_{0}^{*}+K\left(a_{n}^{*} / b_{n}^{*}\right)$ diverges by Stern-Stolz's theorem, cf. [6] and [8]. Hence the left side of (1.3) diverges in the classical sense.

Since $x^{n} \rightarrow 0$, we see that the right side of (1.3) converges by Proposition 1.1 and Worpitzky's theorem (cf. Theorem I.3 in [8]). Hence the left side of (1.3) converges in the modified sense.

It follows from Theorem 1.5 that the following results are obvious. 
Corollary 2.1. For $|q|<1$, the continued fraction $k_{1} q+K\left(k_{2}^{2} / k_{1} q^{2 n+1}\right)$ converges in the modified sense, and

$k_{1} q+\frac{k_{2}^{2}}{k_{1} q^{3}}+\frac{k_{2}^{2}}{k_{1} q^{5}}+\frac{k_{2}^{2}}{k_{1} q^{7}}+\ldots=k_{2}+\frac{k_{1} k_{2} q}{k_{2}}+\frac{k_{2}^{2} q^{2}}{k_{2}}+\frac{k_{1} k_{2} q^{3}}{k_{2}}+\frac{k_{2}^{2} q^{4}}{k_{2}}+\ldots$ (m.c.), where $k_{1}$ and $k_{2}$ are two non-zero constants if $q \neq 0$.

Proof. We can get (2.2) from Theorem 1.5 by putting $a_{0}=q$ and $d=2$.

Remark 2.2. We can get (1.2) from (2.2) by putting $k_{1}=1$ and $k_{2}=1$.

Corollary 2.3. For $|q|<1$, the continued fraction $k q+K\left(1 / k q^{2 n+1}\right)$ converges in the modified sense, and

$$
k q+\frac{1}{k q^{3}}+\frac{1}{k q^{5}}+\frac{1}{k q^{7}}+\cdots=1+\frac{k q}{1}+\frac{q^{2}}{1}+\frac{k q^{3}}{1}+\frac{q^{4}}{1}+\ldots \text { (m.c.) }
$$

where $k$ is a non-zero constant.

Proof. We can get (2.3) from Theorem 1.5 by putting $a_{0}=q, d=2, k_{1}=k$ and $k_{2}=1$.

Corollary 2.4. For $|q|<1$, the continued fraction $q+K\left(k^{2} / q^{2 n+1}\right)$ converges in the modified sense, and

$$
q+\frac{k^{2}}{q^{3}}+\frac{k^{2}}{q^{5}}+\frac{k^{2}}{q^{7}}+\cdots=k+\frac{k q}{k}+\frac{k^{2} q^{2}}{k}+\frac{k q^{3}}{k}+\frac{k^{2} q^{4}}{k}+\cdots \quad \text { (m.c.) }
$$

where $k$ is a non-zero constant.

Proof. We can get (2.4) from Theorem 1.5 by putting $a_{0}=q, d=2, k_{1}=1$ and $k_{2}=k$.

\section{The proofs of Theorems 1.7, 1.8 and their corollaries}

Proof of Theorem 1.7. For convenience, we denote the left side of (1.4) by $r(q)$. That is

Set

$$
r(q)=1-\frac{q}{1}+\frac{q}{1}-\frac{q^{2}}{1}+\frac{q^{2}}{1}-\frac{q^{3}}{1}+\frac{q^{3}}{1}-\ldots
$$

Then

$$
f(q, a)=1-\frac{q}{1}+\frac{q}{a}
$$

Hence

$$
f(q, a)=\frac{a+q-a q}{a+q} \text { and } 1-f(q, a)=\frac{a q}{a+q}
$$

$$
\frac{1-f(q, a)}{f(q, a)}=\frac{a q}{a+q-a q}=\frac{q}{1+q \frac{1-a}{a}}=\frac{q}{1}+\frac{q(1-a)}{a} .
$$


Let $F(q, A)=r(q)$ with

$$
F(q, A)=1-\frac{q}{1}+\frac{q}{A} .
$$

Then (3.1), (3.2) and (3.3) imply that

$$
F(q, A)=\frac{A+q-A q}{A+q}=\frac{1}{1}+\frac{q}{1}+\frac{q(1-A)}{A} .
$$
that

By replacing $A$ on the right side of (3.4) by $f\left(q^{2}, a\right)$ and using (3.3), we get

$$
F(q, A)=\frac{1}{1}+\frac{q}{1}+\frac{q^{3}}{1}+\frac{q^{2}(1-a)}{a} .
$$

We replace $a$ in $(3.5)$ by $f\left(q^{3}, a\right), \ldots$ By repeating this procedure we see that the $2 n$th approximant of the left hand side of (1.4) is equal to the $n$th approximant of the right hand side. Since $q^{n} \rightarrow 0$, we know that two sides of (1.4) converge by Worpitzky's theorem (cf. Theorem I.3 in [8]). Hence the limit of the sequence of the $2 n$th approximants of the left hand side of (1.4) is equal to the one of the sequence of the $n$th approximants of the left hand side. Therefore (1.4) is true.

The following equality follows from the similar arguments as in the proof of Theorem 1.7.

Corollary 3.1. For $|q|<1$,

$$
\begin{aligned}
& 1-\frac{a q}{1}+\frac{a q}{1}-\frac{a q^{k+1}}{1}+\frac{a q^{k+1}}{1}-\frac{a q^{2 k+1}}{1}+\frac{a q^{2 k+1}}{1}-\cdots \\
= & \frac{1}{1}+\frac{a q}{1}+\frac{a^{2} q^{k+2}}{1}+\frac{a^{2} q^{3 k+2}}{1}+\frac{a^{2} q^{5 k+2}}{1}+\cdots
\end{aligned}
$$

where $k=1,2,3, \ldots$ and $a$ is a constant.

By Corollary 3.1, we can easily get the following two equalities.

Corollary 3.2. For $|q|<1$,

$$
1-\frac{q}{1}+\frac{q}{1}-\frac{q^{3}}{1}+\frac{q^{3}}{1}-\frac{q^{5}}{1}+\frac{q^{5}}{1}-\ldots=\frac{1}{1}+\frac{q}{1}+\frac{q^{4}}{1}+\frac{q^{8}}{1}+\frac{q^{12}}{1}+\ldots
$$

Corollary 3.3. For $|q|<1$,

$$
\begin{aligned}
& 1-\frac{q}{1}+\frac{q}{1}-\frac{q^{k+1}}{1}+\frac{q^{k+1}}{1}-\frac{q^{2 k+1}}{1}+\frac{q^{2 k+1}}{1}-\cdots \\
= & \frac{1}{1}+\frac{q}{1}+\frac{q^{k+2}}{1}+\frac{q^{3 k+2}}{1}+\frac{q^{5 k+2}}{1}+\ldots
\end{aligned}
$$

where $k=1,2,3, \ldots$ 
Proof of Theorem 1.8. For convenience, we denote the left side of the equality (1.5) by $B(q)$. That is

$$
B(q)=1+\frac{q}{1}-\frac{q}{1}+\frac{q^{2}}{1}-\frac{q^{2}}{1}+\frac{q^{3}}{1}-\frac{q^{3}}{1}+\ldots
$$

Set

$$
g(q, a)=1+\frac{q}{1}-\frac{q}{a}
$$

Then

$$
g(q, a)=\frac{a-q+a q}{a-q} \text { and } g(q, a)-1=\frac{a q}{a-q}
$$

Hence

$$
\frac{g(q, a)-1}{g(q, a)}=\frac{a q}{a-q+a q}=\frac{q}{1+q \frac{a-1}{a}}=\frac{q}{1}+\frac{q(a-1)}{a} .
$$

Let $G(q, A)=B(q)$ with

$$
G(q, A)=1+\frac{q}{1}-\frac{q}{A} .
$$

Then (3.6), (3.7) and (3.8) imply that

$$
G(q, A)=\frac{A-q+A q}{A-q}=\frac{1}{1}-\frac{q}{1}+\frac{q(A-1)}{A} .
$$

By replacing $A$ on the right side of $(3.9)$ by $g\left(q^{2}, a\right)$ and using $(3.8)$, we get that

$$
G(q, A)=\frac{1}{1}-\frac{q}{1}+\frac{q^{3}}{1}+\frac{q^{2}(a-1)}{a} .
$$

We replace $a$ in $(3.10)$ by $f\left(q^{3}, a\right), \ldots$ By repeating the procedure as above we see that the $2 n$th approximant of the left hand side of (1.5) is equal to the $n$th approximant of the right hand side. Since $q^{n} \rightarrow 0$, we see that two sides of (1.5) converge by Worpitzky's theorem (cf. Theorem I.3 in [8]). Hence the limit of the sequence of the $2 n$th approximants of the left hand side of (1.5) is equal to the one of the sequence of the $n$th approximants of the right hand side. Therefore (1.5) is true.

It follows from similar arguments in Theorem 1.8 that we can get the following.

Corollary 3.4. For $|q|<1$,

$$
\begin{aligned}
& 1+\frac{a q}{1}-\frac{a q}{1}+\frac{a q^{k+1}}{1}-\frac{a q^{k+1}}{1}+\frac{a q^{2 k+1}}{1}-\frac{a q^{2 k+1}}{1}+\cdots \\
= & \frac{1}{1}-\frac{a q}{1}+\frac{a^{2} q^{k+2}}{1}+\frac{a^{2} q^{3 k+2}}{1}+\frac{a^{2} q^{5 k+2}}{1}+\cdots
\end{aligned}
$$

where $k=1,2,3, \ldots$ and $a$ is a constant. 
By Corollary 3.4, we can easily get the following two equalities.

Corollary 3.5. For $|q|<1$,

$$
1+\frac{q}{1}-\frac{q}{1}+\frac{q^{3}}{1}-\frac{q^{3}}{1}+\frac{q^{5}}{1}-\frac{q^{5}}{1}+\ldots=\frac{1}{1}-\frac{q}{1}+\frac{q^{4}}{1}+\frac{q^{8}}{1}+\frac{q^{12}}{1}+\cdots
$$

Corollary 3.6. For $|q|<1$,

$$
\begin{aligned}
& 1+\frac{q}{1}-\frac{q}{1}+\frac{q^{k+1}}{1}-\frac{q^{k+1}}{1}+\frac{q^{2 k+1}}{1}-\frac{q^{2 k+1}}{1}+\cdots \\
= & \frac{1}{1}-\frac{q}{1}+\frac{q^{k+2}}{1}+\frac{q^{3 k+2}}{1}+\frac{q^{5 k+2}}{1}+\ldots
\end{aligned}
$$

where $k=1,2,3, \ldots$.

The proof of Corollary 1.9. We need only to prove the equality (1.6). The proofs of the equalities (1.7), (1.8) and (1.9) follow from similar reasoning. By Theorem 1.7, we have

$$
\frac{q}{1}+\frac{q^{3}}{1}+\frac{q^{5}}{1}+\frac{q^{7}}{1}+\ldots=-1+\frac{1}{1}-\frac{q}{1}+\frac{q}{1}-\frac{q^{2}}{1}+\frac{q^{2}}{1}-\frac{q^{3}}{1}+\frac{q^{3}}{1}-\ldots
$$

By Theorem 1.8, we have

$$
\frac{q}{1}+\frac{q^{3}}{1}+\frac{q^{5}}{1}+\frac{q^{7}}{1}+\cdots=1-\frac{1}{1}+\frac{q}{1}-\frac{q}{1}+\frac{q^{2}}{1}-\frac{q^{2}}{1}+\frac{q^{3}}{1}-\frac{q^{3}}{1}+\ldots .
$$

The equality (1.6) follows from (3.11) and (3.12).

\section{The proof of Theorem $\mathbf{1 . 1 0}$}

Proof of Theorem 1.10. We shall prove the equality (1.12) from the left side to the right side by using the Bauer-Muir transformation. We denote the left side of (1.12) by $B(x)$. That is

$$
B(x)=k_{1}+\frac{k_{2}+a_{1}}{k_{1}}+\frac{k_{2}+a_{2}}{k_{1}}+\cdots .
$$

We choose the modifying factors for $B(x)$ as follows:

$$
\omega_{i}^{(0)}=\beta, \quad i=0,1,2, \ldots
$$

Since

$$
\lambda_{i}^{(0)}=a_{1} x^{(i-1) d}=a_{i} \neq 0, \quad i=1,2,3, \ldots,
$$

it follows from the Bauer-Muir transformation that

$$
\begin{aligned}
B(x) & =k_{1}+\frac{k_{2}+a_{1}}{k_{1}}+\frac{k_{2}+a_{2}}{k_{1}}+\cdots \\
& =k_{1}+\beta+\frac{a_{1}}{k_{1}+\beta}+\frac{\left(k_{2}+a_{1}\right) x^{d}}{k_{1}+\beta-\beta x^{d}}+\frac{\left(k_{2}+a_{2}\right) x^{d}}{k_{1}+\beta-\beta x^{d}}+\cdots \\
& =\alpha+\frac{a_{1}}{B_{1}(x)},
\end{aligned}
$$


where

$$
B_{1}(x)=\alpha+\frac{k_{2} x^{d}+a_{2}}{\alpha-\beta x^{d}}+\frac{k_{2} x^{d}+a_{3}}{\alpha-\beta x^{d}}+\cdots .
$$

We choose the modifying factors for $B_{1}(x)$ as follows:

$$
\omega_{i}^{(1)}=\beta x^{d}, \quad i=0,1,2, \ldots
$$

Then $\lambda_{i}^{(1)}=a_{i+1} \neq 0(i=1,2,3, \ldots)$. The Bauer-Muir transformation yields that

$$
\begin{aligned}
B_{1}(x) & =\alpha+\beta x^{d}+\frac{a_{2}}{\alpha-\beta x^{d}+\beta x^{d}}+\frac{\left(k_{2} x^{d}+a_{2}\right) x^{d}}{\alpha-\beta x^{2 d}}+\frac{\left(k_{2} x^{d}+a_{3}\right) x^{d}}{\alpha-\beta x^{2 d}}+\cdots \\
& =\alpha+\beta x^{d}+\frac{a_{2}}{B_{2}(x)}
\end{aligned}
$$

where

$$
B_{2}(x)=\alpha+\frac{k_{2} x^{2 d}+a_{3}}{\alpha-\beta x^{2 d}}+\frac{k_{2} x^{2 d}+a_{4}}{\alpha-\beta x^{2 d}}+\ldots .
$$

By taking $\omega_{i}^{(k)}=\beta x^{k d}(i \geq 0)$ we see that $\lambda_{i}^{(k)}=a_{i+k} \neq 0(i \geq 1)$ for any $k \geq 0$. The equality (1.12) follows.

Acknowledgement. The authors thank the referee for the careful reading of this paper and many valuable suggestions.

The second author also thanks the support from the project "The program for Science and Technology Innovative Research Team in Higher Educational Institutions of Hunan Province".

\section{References}

[1] K. Alladi, On the modified convergence of some continued fractions of RogersRamanujan type, J. Combin. Theory Ser. A 65 (1994), no. 2, 214-245.

$[2]$ B. C. Berndt, H. H. Chan, S. S. Huang, S. Y. Kang, J. Sohn, and S. H. Son, The Rogers-Ramanujan continued fractions, J. Comput. Appl. Math. 105 (1999), no. 1-2, $9-24$.

[3] B. C. Berndt, S. S. Huang, J. Sohn, and S. H. Son, Some theorem on the RogersRamanujan continued fraction in Ramanujan's lost notebook, Trans. Amer. Math. Soc. 352 (2000), no. 5, 2157-2177.

[4] B. C. Berndt and A. J. Yee, On the generalized Rogers-Ramanujan continued fraction, Ramanujan J. 7 (2003), no. 1-3, 321-331.

[5] L. Jacobsen, On the Bauer-Muir transformation for continued fractions and its applications, J. Math. Anal. Appl. 152 (1990), no. 2, 496-514.

[6] W. B. Jones and W. J. Thron, Continued Fractions: Analytic Theory and Applications, Encyclopedia of Mathematics and its Applications, Addison-Wesley, 1980.

[7] J. Lee and J. Sohn, Modified convergence of Ramanujan type continued fractions, To appear.

[8] L. Lorentzen and H. Waadeland, Continued Fractions with Applications, Studies in Computational Mathematics 3, North-Holland, 1992.

[9] S. Ramanujan, The Lost Notebook and Other Unpublished Papers, Narosa, New Delhi, 1988. 
[10] W. J. Thron and H. Waadeland, Modifications of continued fractions, a survey, Analytic theory of continued fractions (Loen, 1981), pp. 38-66, Lecture Notes in Math., 932, Springer, Berlin-New York, 1982.

\section{YONGQUN Li}

Department of Mathematics

HUNAN UNIVERSITY

Changsha, Hunan 410082, P. R. China

E-mail address: yqlixq@yahoo.com.cn

\section{Xiantao Wang}

Department of Mathematics

Hunan Normal University

Changsha, Hunan 410081, P. R. China

E-mail address: xtwang@hunnu.edu.cn 